\title{
Comparison of skin autofluorescence, a marker of tissue advanced glycation end- products in peritoneal dialysis patients using standard and biocompatible glucose containing peritoneal dialysates
}

\author{
${ }^{1}$ Surachet Vongsanim MD, ${ }^{2}$ Stanley Fan FRCP, ${ }^{3}$ Andrew Davenport FRCP \\ ${ }^{1}$ Renal Division, Department of Internal Medicine, Faculty of Medicine, Chiang Mai University, Suthep \\ Road, Chiang Mai, Thailand \\ ${ }^{2}$ Department of Renal Medicine, Royal London Hospital, Barts Health NHS Trust, Whitechapel Road, \\ London E1 1BB \\ ${ }^{3}$ UCL Department of Nephrology, Royal Free Hospital, University College London, \\ Rowland Hill Street, London NW3 2PF
}

Address for correspondence

Surachet Vongsanim

Surachet.vongsanim@nhs.net

Stanley Fan

fan.stanley@bartshealth.nhs.uk

Andrew Davenport

andrewdavenport@nhs.net

contact andrewdavenport@nhs.net

UCL Department of Nephrology, Royal Free Hospital, University College London,

Rowland Hill Street, London NW3 2PF, ORCID 0000-0002-4467-6833

tel $\quad 44-2074726457$ fax $\quad 44-2073178591$

short title skin autofluorescence and biocompatible peritoneal dialysates

key words peritoneal dialysis skin autofluorescence dual-chamber advanced glycosylation end product bio-compatible dialysate

Funding: None

This article has been accepted for publication and undergone full peer review but has not been through the copyediting, typesetting, pagination and proofreading process, which may lead to differences between this version and the Version of Record. Please cite this article as doi: 10.1111/nep.13510 
Dr Vongsanim was in receipt of a training scholarship from the International Society of Nephrology and Kidney research UK.

\section{Abstract \\ Background}

Heat sterilisation of peritoneal dialysis (PD) dialysates leads to the generation of advanced glycation products (AGEs), which can then deposit in the skin and be measured by skin autofluorescence (SAF). Newer biocompatible dual chamber dialysates contain less AGEs. We wished to determine whether the use of these newer dialysates resulted in lower SAF.

\section{Methods}

SAF was measured using the AGE reader, which directs ultraviolet light, intensity range 300-420 nm (peak $370 \mathrm{~nm}$ ) in patients established on PD for $>3$ months using glucose containing dialysates.

Results

We screened 196 consecutive patients, and measured SAF in 150; 86(57.3\%) male, median age 62 (53-71) years, median duration of PD treatment 17 (8.6-34.3) months. The median SAF was 3.48 (2.92-4.26) AU. The median SAF in the 57 (38\%) patients prescribed biocompatible dual chamber bag dialysates was 3.39 (2.69-3.98) vs 3.5 (3.05-4.54) for those using standard dialysates $(\mathrm{p}=0.044)$. Although prescription of biocompatible fluids was associated with SAF on univariate analysis, but not on multivariable testing, SAF was independently associated with Stoke-Davies co-morbidity grade ( $\beta$ 0.045, 95\% confidence limits (CL) 0.015 to $0.075, \mathrm{p}=0.002$ ), $\log$ duration of PD therapy ( $\beta$ 0.051, CL 0.001 to $0.101, \mathrm{p}=0.045$ ), white ethnicity ( $\beta$ 0.066, CL 0.028 to $0.104, p=0.001$ ), and negatively with serum albumin $(\beta-0.006, \mathrm{CL}$ -0.008 to $-0.004, p=0.014)$

\section{Conclusions}

Although SAF was lower in PD patients prescribed biocompatible dual chamber dialysates, on multivariable testing these dialysates were not independently associated with SAF. Other factors than PD fluid AGE content appear more important in determining SAF.

\section{$\underline{\text { Introduction }}$}

Cardiovascular disease remains the leading cause of death for patients with chronic kidney disease and in particular for those with end-stage kidney disease (ESKD) despite advances in dialysis treatments. ${ }^{(1,2)}$ In addition to the traditional risk factors for cardiovascular disease, dialysis patients also 
have additional non-traditional risk factors, including bone mineral disorders, anaemia, uraemic toxins and oxidative stress. Advanced glycation end products (AGEs) are produced by the non-enzymatic reaction between reducing sugars and protein molecules and are normally cleared by the kidney. Accumulation of these compounds increases oxidative reactions, resulting in inflammation and promoting vascular damage, fibrosis and accelerating atherosclerosis. ${ }^{(3)}$

In the circulation, the plasma concentration of AGEs can vary, falling post-haemodialysis compared to pre-dialysis with high flux and haemodiafiltration treatments and also depending upon the dietary intake of preformed AGEs. ${ }^{(4,5)}$ However increased circulating AGEs results in tissue deposition, and as such tissue deposits more reliably reflect longer term exposure. Skin autofluorescence (SAF) has been shown to be a reliable, non-invasive measurement of the dermal content of AGEs. Previous studies have reported that hemodialysis patients with higher levels of SAF had greater risk of cardiovascular mortality. ${ }^{(6,7)}$

For peritoneal dialysis (PD) patients, glucose is used as the main osmotic agent in dialysate solutions to achieve ultrafiltration. Heat sterilization of these dialysates results in formation of glucose degradation products (GDPs). High level of GDPs in PD dialysate solutions can potentially then lead to increase AGEs in the body. ${ }^{(8,9)}$ Compared to the standard single chamber dialysates, the newer dual chamber peritoneal dialysate bags, which contain glucose in a much greater acidic solution generate much fewer GDPs when sterilised. ${ }^{(10,11)}$ Studies using these newer dual chamber lower GDP dialysates have reported less systemic inflammation. ${ }^{(12)}$ We therefore wished to determine whether treatment of PD patients with these dual chamber lower GDP dialysates, which are marketed as being less bioincompatible reduces tissue AGEs deposition compared to standard dialysates by measuring SAF.

\section{Methods}

We measured SAF when PD patients attended university tertiary centre clinics for routine outpatient review. Patients who had been established on PD for less than 3 months, and those not using glucose containing dialysates were excluded. SAF was measured using AGE reader (DiagnOptics, Groningen, Netherlands), which directs ultraviolet light (UV), intensity range 300-420 nm (peak $370 \mathrm{~nm}$ ), through the skin for excitation AGEs deposited in subcutaneous tissues, and the measured autofluorescence light with a spectrophotometer with a range $300-600 \mathrm{~nm} .{ }^{(13,14)}$ The AGE reader was fitted with the additional light source for those with darker skin pigmentation. SAF was measured three times on the volar surface of the dominant arm, adjusted for skin colour (calculated by AGE reader software), and 
the average value recorded. All measurements were made by the single observer blinded to whether patients used single or dual chamber dialysates.

Peritoneal membrane assessment was by standard methods with peritoneal transporter status calculated from the four-hour peritoneal dialysate effluent creatinine to serum ratio from a peritoneal equilibration test (PET), weekly urea clearance (Kt/Vurea) and dietary protein nitrogen appearance were calculated by standard methods from 24-hour urine and total peritoneal dialysate effluent samples. ${ }^{(15)}$ Glucose absorption was calculated from the amount of glucose in the fresh dialysate and the measured glucose in the spent peritoneal dialysate. No patient had been treated for PD peritonitis or had a hospital admission within preceding 3 months. Patient dialysed using standard glucose dialysates, icodextrin and dual chamber dialysates (Dianeal, Extraneal Baxter Health Care, Deerfield, Illinois, USA and Balance Fresenius Medical Care, Bad Homberg, Germany). Patients had been maintained on either standard glucose dialysates or the newer dual chamber lower GDP dialysates since initiation of PD.

Blood tests were taken concurrently and analysed by standard laboratory methods for urea, creatinine, albumin, haemoglobin, C-reactive protein (CRP), and glycated haemoglobin ${ }^{(16)}$ Relevant medical history and medications were obtained from hospital computerised records. Patients co-morbidity was assessed using the Davies-Stoke co-morbidity score system. ${ }^{(17)}$ Dietary and smoking history were taken by direct patient questioning.

The observational study was registered with National Health Service (NHS) ethics (IRAS129559) and approved by London Camden and Islington research ethics committee (13/LO/0912) and registered (ISRCTN70556765). In keeping with the declaration of Helsinki, patients were required to provide informed consent.

\section{Statistical analysis}

All categorical data are reported as numbers (percentage) and continuous data are presented as mean \pm standard deviation or median (interquartile range). Standard statistical tests were used to analyse data (D'Agostino \& Pearson normality test, t-test, Mann Whitney U test, Chi square test) with appropriate corrections made for multiple testing. Univariate analysis was by Spearman correlation testing. All variables which were significant on univariate analysis and also those up to $\mathrm{p}<0.1$ were then entered into a multivariable step-backward model, with non-parametric variables log transformed if required to improve variable distribution. Variables were then only retained where the $95 \%$ confidence intervals for the estimate did not include zero or there was an improvement in model fit (as demonstrated by the $-2 \log$ likelihood), models were checked for collinearity and variable inflation factor. Statistical analysis used 
Statistical Package for Social Science version 24.0 (IBM Corporation, Armonk, New York, USA). Statistical significance was taken as $\mathrm{p}<0.05$.

\section{Results}

One hundred and ninety-six consecutive PD patients, established on PD for more than 3 months using glucose containing dialysates attended the univeristy PD clinics. We were able to measure SAF in 150 patients; 86(57.3\%) male, median age 62 (53-71) years, median duration of PD treatment 17 (8.634.3) months. Six (4\%) patients were vegetarian. The median SAF was 3.48 (2.92-4.26) AU. We were unable to measure SAF in 46 patients, $28(60.9 \%)$ male, mean age $56.5 \pm 15$ years, $28(60.9 \%)$ were African or Afro-Caribbean and 14 (30.4\%) South Asian. We were able to measure SAF in all White and East Asian patients, $82.3 \%$ of South Asian and $22.2 \%$ of African or Afro-Caribbean.

Fifty-seven (38\%) of the patients with SAF measurement were prescribed dual chamber lower GDP dialysates (Table 1). There was no difference in patient ethnicity between those prescribed dual chamber lower GDP dialysates, either when comparing the different ethnicities ( $\mathrm{X} 2=1.83, \mathrm{p}=0.87)$, or as white vs non-white ethnicity $(\mathrm{X} 2=0.16, \mathrm{p}=0.89)$. The SAF level was lower in the patients using dual chamber lower GDP dialysates comparing to the ones using standard dialysates (Figure 1). SAF was lower, but not statistically different, in those non-white patients using dual chamber lower GDP dialysates ( $3.21 \pm 1.06$ vs $3.46 \pm 0.77 \mathrm{AU})$, and also white patients ( $4.05 \pm 1.06$ vs $4.08 \pm 1.17 \mathrm{AU})$. Patients using dual chamber lower GDP dialysates used less icodextrin, had dialysed for a shorter period and had greater proportion were non-smokers. There were no differences in sex, age, race, residual renal function, total weekly Kt/Vurea, peritoneal transporter status, peritoneal dialysis modality, glycated haemoglobin, nPNA, diabetes or co-morbidity score between groups.

We then divided patients according to the median SAF of $3.84 \mathrm{AU}$. There was no difference in patient age, proportion of patients with diabetes, or glycated haemoglobin, or prescription of dextrose dialysates or icodextrin (table 2). However, those patients with higher SAF had lower residual renal function and had been treated by PD for longer.

On univariate analysis SAF was associated with white ethnicity, $\mathrm{C}$ reactive protein, diabetes, comorbidity score and longer treatment with PD and negatively with residual renal function, serum albumin, nPNA and use of icodextrin and dual chamber glucose dialysates (table 3).

We then analysed a multivariable model, and SAF was independently associated with white ethnicity, co-morbidity grade, and longer treatment with PD, and negatively with serum albumin (table 4). 


\section{Discussion}

Heating glucose in solution leads to the formation of 3 de-oxyglucasone and other glucose degradation products (GDPs) which promote the formation of AGEs. ${ }^{(8)}$ Alternative methods of sterilisation, such as filtration reduce the amounts of GDPs in peritoneal dialysates. ${ }^{(1)}$ Nevertheless heat sterilisation is currently the only method permitted for PD dialysates. However, the amount of GDPs generated by heat sterilisation can be reduced when glucose is heated in an increasingly acidic solution. ${ }^{(1)}$ As such the amount of GDPs are much lower in dual chamber PD dialysates compared to standard single chamber dialysates. ${ }^{(10)}$ Clinical studies have observed that GDPs from the PD dialysates can be transported across the peritoneal membrane, and be detected in serum. ${ }^{(9)}$ Other studies have reported that patients using dual chamber PD lower GDP dialysates have lower levels of systemic inflammation. ${ }^{(12)}$ Circulating AGEs lead to skin deposition, and studies have shown a strong association between skin AGE deposition and SAF. ${ }^{(13)}$ As a number of observational studies have reported an association between SAF and mortality in haemodialysis patients we wished to determine whether the use of dual chamber lower GDP PD dialysates would result in lower SAF. ${ }^{(6,7)}$

We found that SAF was significantly lower in PD patients using dual chamber lower GDP PD dialysates compared to standard single chamber glucose dialysates. Patients prescribed the dual chamber lower GDP PD dialysates had borderline lower $\mathrm{C}$ reactive protein and greater serum albumin compared to those using standard PD dialysates, which would be in keeping with AGEs as biomarkers of tissue stress.

However, patients prescribed dual chamber lower GDP PD dialysates had dialysed for a shorter time period and a greater proportion were non-smokers, and fewer used icodextrin. It is unclear as to whether SAF continues to accumulate with time in PD patients. Importantly there was no difference in residual renal function, PD modality or peritoneal transporter status on multivariate analysis . Studies in haemodialysis patients have reported an increase in patients treated by haemodialysis, but not for those treated by haemodiafiltration. ${ }^{(14)}$ As if the production of AGEs is matched by the additional removal of AGEs with convective clearance during haemodialfiltration, then levels would not be expected to increase, whereas depending upon the dialyzer membrane pore size, then AGEs could accumulate in haemodialysis patients, once residual renal function had been lost. ${ }^{(18)}$ Although some previous reports have demonstrated an association between smoking status and SAF, ${ }^{(19)}$ we were unable to demonstrate an association. 
A previous report has suggested that the use of icodextrin dialysates may lead to an increase in SAF ${ }^{(20)}$ Our patients prescribed dual chamber lower GDP dialysates had lower SAF, but they also had lower icodextrin usage. Although icodextrin usage could have potentially introduced bias, when we analysed patients according to SAF, there was no effect of icodextrin.

On univariate analysis, as expected we found a negative association between residual renal urea clearance and $\mathrm{SAF}$, and lower SAF was also associated with lower co-morbidity, lower $\mathrm{C}$ reactive protein, higher serum albumin, greater nPNA and use of dual chamber lower GDP dialysates. Although previous reports have suggested that lower protein diets may lead to lower SAF, by reducing dietary preformed AGEs, we suspect that the association with nPNA is linked to healthier fitter patients generating more urea nitrogen, as more active life-styles are recognised to reduce SAF. ${ }^{(5,21,22)}$ On the other hand increasing SAF was associated with increasing co-morbidity, diabetes and longer duration of PD therapy. Some previous reports have observed an association with diabetes, although this has not been universal. ${ }^{(6,23,24)}$ In our series we measured glycated haemoglobin in all patients and found no association between glycated haemoglobin and SAF. As we report an observational study we were unable to determine whether the increasing SAF with longer duration of PD therapy was due to greater exposure to PD dialysates, or loss of residual renal function.

SAF was greater in patients of white ethnicity. Although the AGE reader is fitted with an additional light source to allow for measurements of SAF in patients with darker skin pigmentation, we were unable to measure SAF in around $17 \%$ of South Asian and $78 \%$ of African-Afro-Caribbean patients due to very dark skin pigmentation. As there was no difference in the proportion of ethnic minorities between the groups we do not think that this would have biased our results. Some studies have previously reported a lower success rate in measuring SAF in patients with heavily pigmented skin, although others have reported successfully measuring SAF in African-Americans with sickle cell disease, and patients from sub-Saharan Africa. ${ }^{(25-27)}$ SAF is associated with increased mortality for both haemodialysis and PD patients, ${ }^{(7,28)}$ and observational studies from North America, United Kingdom and Demark all report increased mortality for white dialysis patients compared to those from the ethnic minorities. ${ }^{(29-31)}$

We report an observational study, and although there was a difference in SAF between those patients prescribed dual chamber lower GDP glucose containing dialysates, on multivariable testing, this difference no longer remained statistically significant. Previous reports have noted that prescription of these dialysates leads to a reduction in biomarkers of systemic inflammation, and endothelial dysfunction, ${ }^{(12)}$ and others lower peritoneal effluent cancer antigen 125 and ultrafiltration volumes, suggestive of less intra-peritoneal inflammation. ${ }^{(32)}$ Our patients prescribed dual chamber lower GDP PD 
dialysates had marginally higher serum albumin and lower $\mathrm{C}$ reactive protein levels, and we are unable to determine whether this was consequent upon the dialysate prescribed. In addition, the patients treated by dual chamber lower GDP PD dialysates had been treated with PD for a shorter PD time, although residual urinary urea clearances were not different.

Our study would suggest that although the use of dual chamber lower GDP PD dialysates may appear to lead to lower accumulation of AGEs resulting in lower SAF, we found that in our crosssectional study that other factors than the choice of PD dialysate were more important in determining $\mathrm{SAF}$, such as duration of PD treatment, residual renal function, patient co-morbidity and serum albumin. 
Fig. 1 Difference in skin autofluorescence (SAF) between using standard dialysates and dual chamber lower glucose degradation product dialysates in peritoneal dialysis patients. $* \mathrm{p}<0.05$

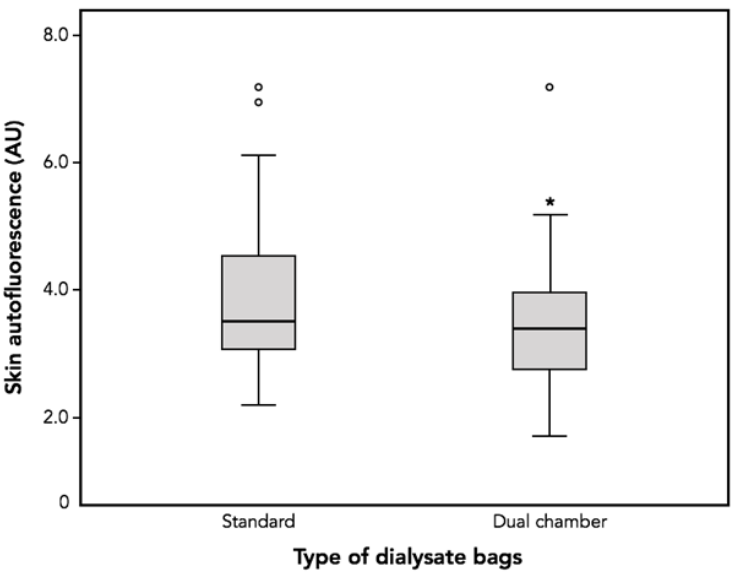


Table 1 Patients demographic using dual chamber bag low glucose degradation product (GDP) dialysates compared to those using single bag dialysates. Values presented as percentage, mean \pm standard deviation or median (interquartile range). Body mass index (BMI), peritoneal equilibration test (PET), dialysate (D), plasma (P), automated peritoneal dialysis cycler (APD), continuous ambulatory peritoneal dialysis (CAPD), glycated haemoglobin (HbA1C), C-reactive protein (CRP), normalised protein nitrogen appearance rate (nPNA). Results as integer, percentage, mean \pm standard deviation or median (interquartile range). $\mathrm{p}$ values vs single chamber glucose dialysate group.

\begin{tabular}{|c|c|c|c|c|}
\hline Variables & Overall $(\mathrm{N}=150)$ & Single bag $(\mathrm{N}=93)$ & Dual chamber low GDP(N=57) & P-value \\
\hline Male (\%) & $86(57.3)$ & $53(57.0)$ & $33(57.9)$ & 0.913 \\
\hline Age, years & $62(53-71)$ & $63.5(55.3-73.7)$ & $59(50.5-69.0)$ & 0.240 \\
\hline Smoking status (\%) & & & & 0.002 \\
\hline - Never & $102(69.4)$ & $54(60)$ & $48(84.2)$ & \\
\hline - Ex-smoker & $17(11.6)$ & $11(12.2)$ & $6(10.5)$ & \\
\hline - Current smoker & $28(19)$ & $25(27.8)$ & $3(5.3)$ & \\
\hline Race (\%) & & & & 0.131 \\
\hline - White & $63(42)$ & $44(47.3)$ & $19(33.3)$ & \\
\hline - South Asian & $65(43.3)$ & $36(38.7)$ & $29(50.9)$ & \\
\hline - Others & $22(14.7)$ & $15(14)$ & $9(14.1)$ & \\
\hline BMI & $27.1 \pm 4.9$ & $26.5 \pm 4.79$ & $28.2 \pm 5.11$ & 0.147 \\
\hline DM (\%) & $59(39.3)$ & $38(40.9)$ & $21(36.8)$ & 0.625 \\
\hline Davies comorbidity (\%) & & & & 0.108 \\
\hline - Grade 0 & $56(37.3)$ & $29(31.2)$ & $27(47.4)$ & \\
\hline - Grade 1 & $79(52.7)$ & $55(59.1)$ & $24(42.1)$ & \\
\hline - Grade 2 & $15(10)$ & $9(9.7)$ & $6(10.5)$ & \\
\hline Icodextrin usage (\%) & $103(68.7)$ & $70(75.3)$ & $33(57.9)$ & 0.026 \\
\hline Dialysis vintage, months & $17(8.62-34.25)$ & $21.5(12-38.75)$ & $14.04(4.58-31.96)$ & 0.007 \\
\hline \multicolumn{5}{|l|}{ Weekly Kt/Vurea } \\
\hline - Total & $1.92(1.55-2.42)$ & $1.92(1.59-2.44)$ & $2.06(1.50-2.42)$ & 0.593 \\
\hline - residual Renal Kt/Vurea & $0.57(0.10-1.25)$ & $0.45(0.08-0.96)$ & $0.79(0.17-1.33)$ & 0.072 \\
\hline - peritoneal Dialysis Kt/Vurea & $1.30(1.00-1.59)$ & $1.37(1.12-1.63)$ & $1.23(0.86-1.44)$ & 0.001 \\
\hline Urine volume, $\mathrm{ml} /$ day & $600(344.8-1304.3)$ & $508(100-833.8)$ & $850(312.5-1400)$ & 0.054 \\
\hline PET 4 hr D/Pcreatinine & $0.68 \pm 0.14$ & $0.72 \pm 0.13$ & $0.62 \pm 0.12$ & $<0.001$ \\
\hline Peritoneal Dialysis mode & & & & 0.242 \\
\hline - CAPD (\%) & $16(10.7)$ & $11(11.8)$ & $5(8.8)$ & \\
\hline
\end{tabular}




\begin{tabular}{|l|c|c|c|c|}
\hline - APD dry day (\%) & $41(27.3)$ & $21(22.6)$ & $20(35.1)$ & \\
\hline - APD wet day (\%) & $93(62)$ & $61(65.6)$ & $32(56.1)$ & \\
\hline Serum albumin, g/L & $38.13 \pm 4.3$ & $37.6 \pm 4.01$ & $39 \pm 4.66$ & 0.051 \\
\hline HbA1C, mol/mmol & $40.5(35-55.2)$ & $41(36.6-55.2)$ & $38.5(33-57.25)$ & 0.255 \\
\hline CRP, $\mathrm{mg} / \mathrm{L}$ & $4(2-12)$ & $5(2-16.75)$ & $3.5(1-10)$ & 0.050 \\
\hline nPNA, g/kg/day & $0.92(0.69-1.12)$ & $0.90(0.70-1.12)$ & $0.96(0.69-1.11)$ & 0.668 \\
\hline
\end{tabular}

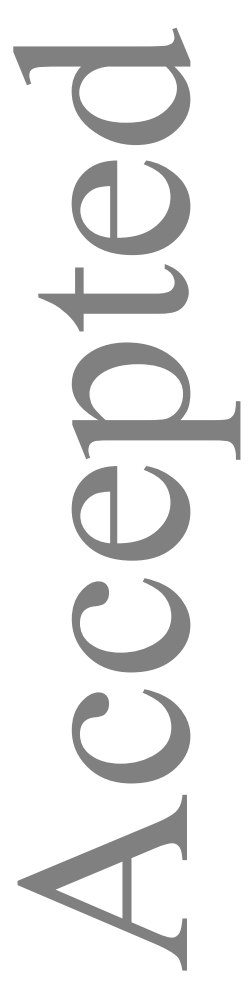


Table 2. Peritoneal Dialysis (PD) patients divided into those with higher and lower skin autofluorescence (SAF). Combined 24 hour urinary urea and creatinine clearance (residual renal function), Glycated haemoglobin (HbAlc). Values as integers (percentage), or median (interquartile range).

\begin{tabular}{|l|l|l|l|}
\hline Variable & Higher SAF & Lower SAF & $\mathrm{p}$ value \\
\hline number & 73 & 77 & \\
\hline SAF AU & $4.27(3.93-4.83)$ & $2.95(2.48-3.27)$ & $<0.001$ \\
\hline Age years & $63(55-71)$ & $61(50-72)$ & $>0.05$ \\
\hline Residual renal function $\mathrm{mL} / \mathrm{min} / 1.73 \mathrm{~m}^{2}$ & $0.7(0.2-2.0)$ & $1.4(0.5-2.7)$ & 0.039 \\
\hline PD vintage months & $22.7(12.1-41.2)$ & $12.1(5.4-31.4)$ & 0.001 \\
\hline Glucose exposure g/day & $146(106-204)$ & $129(98-182)$ & $>0.5$ \\
\hline Diabetic $(\%)$ & $31(42.6)$ & $28(36.4)$ & $>0.05$ \\
\hline HbA1c mol/mmol & $41(35.5-55.2)$ & $39.9(34.4-56.1)$ & $>0.05$ \\
\hline Icodextrin prescribed (\%) & $50(68.5)$ & $53(68.3)$ & $>0.05$ \\
\hline
\end{tabular}


Table 3 Spearman univariate analysis for factors associated with skin autofluorescence. Glucose degradation products (GDP).

\begin{tabular}{|l|c|c|}
\hline Variables & $\mathrm{r}$ & P-value \\
\hline White ethnicity & 0.323 & $<0.001$ \\
\hline Smoking status & 0.141 & 0.088 \\
\hline Diabetes mellitus & 0.182 & 0.026 \\
\hline Davies comorbidity grade & 0.260 & 0.001 \\
\hline Months of peritoneal dialysis treatment & 0.298 & $<0.001$ \\
\hline 24 hour urinary Kt/Vurea & -0.221 & 0.008 \\
\hline Serum albumin & -0.375 & $<0.001$ \\
\hline Serum C-reactive protein & 0.278 & 0.001 \\
\hline Normalised protein nitrogen appearance & -0.229 & 0.008 \\
\hline Dual chamber low GDP glucose dialysates & -0.165 & 0.044 \\
\hline
\end{tabular}


Table 4 Multivariable model for $\log$ skin autofluorescence. Standard error $\beta$ (StE $\beta$ ), standardised $\beta$ (St $\beta$ ), $95 \%$ confidence intervals $(95 \% \mathrm{CI})$. Model fit $\mathrm{r}^{2}=0.286$, adjusted $\mathrm{r}^{2}=0.263$.

\begin{tabular}{|l|l|c|c|c|c|c|c|}
\hline & $\beta$ & $\mathrm{StE} \beta$ & $\mathrm{St} \beta$ & $\mathrm{t}$ & $95 \%$ CI & P-value \\
\hline Variables & -0.006 & 0.002 & -0.203 & -2.483 & -0.008 to -0.004 & 0.014 \\
\hline Serum albumin & 0.045 & 0.015 & 0.244 & 3.128 & 0.015 to 0.075 & 0.002 \\
\hline Stoke-Davies grade & 0.066 & 0.019 & 0.277 & 3.471 & 0.028 to 0.104 & 0.001 \\
\hline & White ethnicity & 0.051 & 0.025 & 0.163 & 2.029 & 0.001 to 0.101 & 0.045 \\
\hline
\end{tabular}




\section{References}

1. USRDS Chapter 9: Cardiovascular Disease in Patients With ESRD. . Am J Kidney Dis 2016;67(3):S259-64.

2. Wang IK, Lu CY, Lin CL, Liang CC, Yen TH, Liu YL, et al. Comparison of the risk of de novo cardiovascular disease between hemodialysis and peritoneal dialysis in patients with end-stage renal disease. Int J Cardiol. 2016;218:219-24.

3. Yan SF, Ramasamy R, Naka Y, Schmidt AM. Glycation, inflammation, and RAGE: a scaffold for the macrovascular complications of diabetes and beyond. Circ Res. 2003;93(12):1159-69.

4. Cornelis T, Eloot S, Vanholder R, Glorieux G, van der Sande FM, Scheijen JL, et al. Proteinbound uraemic toxins, dicarbonyl stress and advanced glycation end products in conventional and extended haemodialysis and haemodiafiltration. Nephrol Dial Transplant. 2015;30(8):1395-402.

5. Nongnuch A, Davenport A. The effect of vegetarian diet on skin autofluorescence measurements in haemodialysis patients. Br J Nutr. 2015;113(7):1040-3.

6. Nongnuch A, Davenport A. Skin autofluorescence advanced glycosylation end products as an independent predictor of mortality in high flux haemodialysis and haemodialysis patients. Nephrology (Carlton). 2015;20(11):862-7.

7. Meerwaldt R, Hartog JW, Graaff R, Huisman RJ, Links TP, den Hollander NC, et al. Skin autofluorescence, a measure of cumulative metabolic stress and advanced glycation end products, predicts mortality in hemodialysis patients. J Am Soc Nephrol. 2005;16(12):3687-93.

8. Linden T, Forsback G, Deppisch R, Henle T, Wieslander A. 3-Deoxyglucosone, a promoter of advanced glycation end products in fluids for peritoneal dialysis. Perit Dial Int. 1998;18(3):290-3.

9. Zeier M, Schwenger V, Deppisch R, Haug U, Weigel K, Bahner U, et al. Glucose degradation products in PD fluids: do they disappear from the peritoneal cavity and enter the systemic circulation? Kidney Int. 2003;63(1):298-305.

10. Diaz-Buxo J, Clark SC, Ho CH, Jensen LE. New pH-neutral peritoneal dialysis solution, low in glucose degradation products, in a double-chamber bag. Adv Perit Dial. 2010;26:28-32.

11. Erixon M, Wieslander A, Linden T, Carlsson O, Forsback G, Svensson E, et al. How to avoid glucose degradation products in peritoneal dialysis fluids. Perit Dial Int. 2006;26(4):490-7. 
12. Park SH, Do JY, Kim YH, Lee HY, Kim BS, Shin SK, et al. Effects of neutral pH and lowglucose degradation product-containing peritoneal dialysis fluid on systemic markers of inflammation and endothelial dysfunction: a randomized controlled 1-year follow-up study. Nephrol Dial Transplant. 2012;27(3):1191-9.

13. Meerwaldt R, Graaff R, Oomen PHN, Links TP, Jager JJ, Alderson NL, et al. Simple noninvasive assessment of advanced glycation endproduct accumulation. Diabetologia. 2004;47(7):1324-30. 14. Nongnuch A, Davenport A. The Effect of On-Line Hemodiafiltration, Vegetarian Diet, and Urine Volume on Advanced Glycosylation End Products Measured by Changes in Skin Auto-Fluorescence. Artif Organs. 2018.

15. Peritoneal Dialysis Adequacy Work G. Clinical practice guidelines for peritoneal adequacy, update 2006. Am J Kidney Dis. 2006;48 Suppl 1:S91-7.

16. Persaud J, Thomas M, Davenport A. Indirect ion selective electrode methods potentially overestimate peritoneal dialysate sodium losses. Ther Apher Dial. 2014;18(4):321-5.

17. Davies SJ, Phillips L, Naish PF, Russell GI. Quantifying comorbidity in peritoneal dialysis patients and its relationship to other predictors of survival. Nephrol Dial Transplant. 2002;17(6):1085-92. 18. Davenport A. How can dialyzer designs improve solute clearances for hemodialysis patients? Hemodial Int. 2014;18 Suppl 1:S43-7.

19. van Waateringe RP, Mook-Kanamori MJ, Slagter SN, van der Klauw MM, van VlietOstaptchouk JV, Graaff R, et al. The association between various smoking behaviors, cotinine biomarkers and skin autofluorescence, a marker for advanced glycation end product accumulation. PLoS One. 2017;12(6):e0179330.

20. Mácsai E, Benke A, Cseh A, Vásárhelyi B. Factors influencing skin autofluorescence of patients with peritoneal dialysis. Acta Physiol Hung. 2012 Jun;99(2):216-22

21. El-Kateb S, Sridharan S, Farrington K, Davenport A. Comparison of resting and total energy expenditure in peritoneal dialysis patients and body composition measured by dual-energy X-ray absorptiometry. Eur J Clin Nutr. 2016;70(11):1337-9.

22. Drenth H, Zuidema SU, Krijnen WP, Bautmans I, Smit AJ, Schans CV, et al. Advanced Glycation End-products are associated with Physical Activity and Physical Functioning in the older population. J Gerontol A Biol Sci Med Sci. 2018.

23. Siriopol D, Hogas S, Veisa G, Mititiuc I, Volovat C, Apetrii M, et al. Tissue advanced glycation end products (AGEs), measured by skin autofluorescence, predict mortality in peritoneal dialysis. Int Urol Nephrol. 2015;47(3):563-9. 
24. Oleniuc M, Schiller A, Secara I, Onofriescu M, Hogas S, Apetrii M, et al. Evaluation of advanced glycation end products accumulation, using skin autofluorescence, in CKD and dialysis patients. Int Urol Nephrol. 2012;44(5):1441-9.

25. Chaudhri S, Fan S, Davenport A. Pitfalls in the measurement of skin autofluorescence to determine tissue advanced glycosylation content in haemodialysis patients. Nephrology (Carlton). 2013;18(10):671-5.

26. Kashyap L, Alsaheel A, Ranck M, Gardner R, Maynard J, Chalew SA. Sickle Cell Disease is Associated With Elevated Levels of Skin Advanced Glycation Endproducts. J Pediatr Hematol Oncol. 2018;40(4):285-9.

27. Ahmad MS, Kimhofer T, Ahmad S, AlAma MN, Mosli HH, Hindawi SI, et al. Ethnicity and skin autofluorescence-based risk-engines for cardiovascular disease and diabetes mellitus. PLoS One. 2017;12(9):e0185175.

28. Macsai E, Benke A, Kiss I. Skin Autofluorescence and Mortality in Patients on Peritoneal Dialysis. Medicine (Baltimore). 2015;94(45):e1933.

29. Derose SF, Rutkowski MP, Crooks PW, Shi JM, Wang JQ, Kalantar-Zadeh K, et al. Racial differences in estimated GFR decline, ESRD, and mortality in an integrated health system. Am J Kidney Dis. 2013;62(2):236-44.

30. Roderick P, Byrne C, Casula A, Steenkamp R, Ansell D, Burden R, et al. Survival of patients from South Asian and Black populations starting renal replacement therapy in England and Wales. Nephrol Dial Transplant. 2009;24(12):3774-82.

31. van den Beukel TO, Hommel K, Kamper AL, Heaf JG, Siegert CE, Honig A, et al. Differences in survival on chronic dialysis treatment between ethnic groups in Denmark: a population-wide, national cohort study. Nephrol Dial Transplant. 2016;31(7):1160-7.

32. Cho Y, Johnson DW. Does the use of neutral $\mathrm{pH}$, low glucose degradation product peritoneal dialysis fluids lead to better patient outcomes? Curr Opin Nephrol Hypertens. 2014;23(2):192-7. 